\title{
RE II - ein Briefwechsel
}

\section{FMH}

Dr. H. H. Brunner

Elfenstrasse 18

3000 Bern 16

Zürich, 15. April 2002

\section{RE II}

\section{Sehr geehrter Herr Brunner}

Nach wie vor ist nicht restlos klar, was der genaue Inhalt des RE II ist, welches Prozedere zur Anwendung kommt und wie der genaue Zeitplan aussieht. Gemäss Ihren Aussagen liegt das bereinigte Konzept für das RE II vor, wobei nicht bekannt ist, wer dieses definitiv festgelegt hat. Nach allen bisher gemachten Erfahrungen wäre es wohl zweckmässig gewesen, den (betroffenen) Fachgesellschaften das RE II-Konzept samt Vertrag zur kurzen Vernehmlassung zuzustellen.

Die FMS erwartet, dass das verabschiedete Konzept RE II samt Vertrag so bald wie möglich den FMS-Fachgesellschaften (bitte mit Kopie ans FMS-Sekretariat) zugestellt wird. Wir erinnern auch an den Ärztekammerbeschluss vom 13. Dezember 2001, wonach die Unterlagen für die RE II-Arbeiten ohne Verzug den Delegierten der Ärztekammer zugänglich gemacht werden. Ferner berufen wir uns auf zwei Ärztekammerbeschlüsse vom 12. April 2001 und 13. Dezember 2001, wonach Mängel vor Einführung des Tarifs behoben bzw. sämtliche Datenbanken, die vom RE betroffen sind, der Ärztekammer und der Präsidentenkonferenz zur Verfügung gestellt werden müssen.

Ein besonderes Problem stellt sich aus unserer Sicht hinsichtlich des Timings: Das RE II soll bis 30. Juni 2003 abgeschlossen sein; bis dann wird hoffentlich eine reengineerte Tarifstruktur vorliegen, bei welcher alle jetzt noch vorhandenen schwerwiegenden Mängel beseitigt sind.

Dies bedeutet, dass - nachdem der Bundesrat bzw. das EDI seine «Genehmigung» erteilt hat - diese revidierte Tarifstruktur erst per 1. Januar 2004 wird implementiert und damit in Kraft treten können.

Ist es zweckmässig und vor allem vertretbar, gegebenenfalls am 1. Januar 2003 eine mit noch schwerwiegenden Mängeln behaftete Tarifstruktur in Kraft zu setzen, um ein Jahr später eine revidierte Tarifstruktur erneut in Kraft zu setzen? Wir glauben nicht!
Nachdem mehrere Gross-Spitäler - allen voran das Inselspital - die integrale Einführung von TARMED als Tarif per 1. Januar 2004 sehen, werden wohl per 1. Januar 2003 noch nicht alle Voraussetzungen gegeben sein, um eine möglichst reibungslose Einführung zu gewährleisten. Wir bitten Sie, sich zu dieser nunmehr abzeichnenden Problematik kurz zu äussern.

Eine weitere Frage beschäftigt die FMS:

Vor einigen Tagen haben Sie bekannt gegeben, dass die Ärztekammer die für die Durchführung des RE II notwendigen finanziellen Mittel zu bewilligen habe, «ansonst das Projekt nicht realisierbar sei».

Die FMS vertritt die Auffassung, dass das RE II jedenfalls und ohne weitere Bedingungen durchgeführt werden muss. Wir erachten es als höchst problematisch, wenn gerade ein solches notwendiges Projekt als präjudizierender Einzelfall in finanzieller Hinsicht behandelt wird, zumal die finanzielle Frage unseres Wissens erst heute klar ausgesprochen wird, nachdem die Arbeiten zum RE II teilweise schon angelaufen sind. Die FMS vertritt die Auffassung, dass das RE II aus demselben Budget finanziert werden muss, aus dem bisher alle TARMED-Aktivitäten bestritten wurden. Sind die Mittel zu knapp geworden, dann ist generell (und nicht einzelfallbezogen) eine Aufstockung zu beantragen.

Gerne erwarten wir Ihre baldige Stellungnahme.

Mit bestem Dank und mit freundlichen Grüssen FOEDERATIO MEDICORUM SCRUTANTIUM

\section{Prof. Reto Tscholl, Präsident}

François A. Bernath, Generalsekretär 
FMS

Herrn Prof. R. Tscholl

Herrn F. A. Bemath

Postfach

8034 Zürich

Bern, 28. Mai 2002

\section{RE II - Ihr Schreiben vom 15. April 2002}

Sehr geehrter Herr Tscholl

Sehr geehrter Herr Bernath

Wir nehmen Bezug auf Ihr rubriziertes Schreiben und beantworten Ihnen gerne, soweit möglich, die darin gestellten Fragen. Wir gehen davon aus, dass einige der aufgeworfenen Fragen bereits an der Ärztekammer vom 25. April 2002 geklärt wurden.

Da Ihr Schreiben unseres Erachtens sehr wichtige Fragen aufwirft, die von allgemeinem Interesse sind, würden wir uns ohne ihren Gegenbericht innert fünf Tagen erlauben, dieses sowie unsere Antwort in der Schweizerischen Ärztezeitung zu veröffentlichen.

\section{Inhalt und Zeitplan des RE II, Genehmigung und weiteres Vorgehen}

Das Detailkonzept zum RE II wurde von der Projektleitung TARMED (PL) erstellt und von der Projektoberleitung TARMED (POL) am 11. April 2002 genehmigt. Es besteht aus sieben Teilprojekten, welche skizziert, terminiert und budgetiert wurden. Eine verfeinerte Projektplanung wurde noch nicht erstellt, da zunächst ein Grundsatzentscheid sämtlicher Tarifpartner vorliegen muss. Ärzteseitig ist dieser Entscheid an der Ärztekammer erfolgt: Den Delegierten wurden Konzept und Budget präsentiert und von diesen genehmigt. Die Genehmigung war vonnöten, da der Sonderbeitrag für Tarifentwicklung per 2002 weggefallen ist. Für das Budget 2003 hat die Ärztekammer - unter anderem auch in Anerkennung der Aufwendungen im Zusammenhang mit der Tarifpflege - eine Mitgliederbeitragserhöhung bewilligt. Die TARMED-Projektgremien werden in der Folge über das weitere Vorgehen, die Priorisierung, Ausarbeitung und Umsetzung befinden.

\section{Zur-Verfügung-Stellung von Datenbanken}

Am 13. Dezember 2001 beschloss die Ärztekammer, dass sämtliche Datenbanken den Delegierten der Ärztekammer und der Präsidentenkonferenz offengelegt werden müssen. Die entsprechenden Daten sind bereits seit geraumer Zeit allen berechtigten Mitgliedern auf der geschätzten Website des Tarifdienstes (CUG) zugänglich. Sie werden sukzessive bereinigt und redaktionell aufgearbeitet.

\section{Timing Einführung, RE II, Tarifpflege}

Die Ärzteschaft hat im Rahmen der Urabstimmung der vorliegenden Tarifversion 1.1 als Einführungsversion zugestimmt. Dies nachdem im Rahmen des Reengineering I (RE I) im Jahr 2001 auf Forderung der FMH noch eine Nachbesserung der vorgängigen Tarifstruktur 1.0 erfolgt war, die zum Ziel hatte, Positionen existenzbedrohter Fachgesellschaften zu überarbeiten sowie redaktionelle und regeltechnische Fehler zu beheben. Die Ärzteschaft beschloss ebenfalls die Durchführung eines Reengineerings II (RE II) als «causa sine qua non» zur Zustimmung zur Tarifsstruktur 1.1, welches per Mitte 2003 abgeschlossen sein muss und frühestens auf den ersten Revisionstermin hin wirksam werden kann. In Ihrem Schreiben erwähnen sie den 1. Januar 2004. Dies mag zutreffen, hängt jedoch vom Genehmigungsverfahren des Bundesrates $\mathrm{ab}$, weshalb wir uns hierzu nicht äussern. Es ist jedoch vorgesehen, die Tarifstruktur TARMED jährlich in einem ordentlichen Verfahren zu revidieren, um dem Gebot der Tarifpflege und -wartung Rechnung zu tragen. Die FMH hat bereits Vorkehrungen getroffen, um den Ablauf ärzteseitig zu regeln (vgl. Rundschreiben vom 19. April 2002 inkl. Merkblatt).

\section{Koordination mit dem Spitalbereich}

Wir haben von der Haltung vom Schweizerischen Spitalverband $\mathrm{H}+$ über den aus seiner Sicht frühestmöglichen Einführungstermin Kenntnis erhalten. Für diese Verlautbarung lehnen wir jegliche Verantwortung ab. FMH und G7 setzen alles daran, den Einführungstermin des TARMED für die Abrechnung von Leistungen für Patientinnen und Patienten der obligatorischen Krankenpflegeversicherung in Arztpraxen auf den 1. Januar 2003 einzuhalten. Gleichzeitig bindet uns der von der Ärzteschaft gefällte und wiederholt bekräftigte Beschluss, die gleichen Konditionen wie im Spitalbereich walten zu lassen, was auch für die Frage des Einführungstermins gilt. Wir hoffen, Ihnen mit diesen Auskünften gedient zu haben.

Mit freundlichen Grüssen

(namens der Verhandlungsdelegation)

\section{Annamaria Müller Imboden}

Kopie:

- TAKO 